\title{
Una enfermería del trabajo con perspectiva holística
}

\author{
Occupational health nursing: \\ a holistic perspective
}

Javier González-Caballero' 이 0000-0003-0025-4644

${ }^{1}$ Servicio de Prevención y Salud Laboral. Instituto Nacional de la Seguridad Social. Bilbao, España.

Fechas · Dates

Recibido: 2020.12.23

Aceptado: 2021.02.10

Publicado: 2021.04.15
Correspondencia · Corresponding Author

Javier González-Caballero

Gran Vía don Diego López de Haro, 62. 48011 Bilbao. Bizkaia.

javier.gonzalez1@seg-social.es 


\title{
Resumen
}

La enfermería del trabajo se encuentra en una posición privilegiada para identificar las necesidades de la población laboral en el ámbito de sus competencias. En los distintos escenarios posibles del futuro de la actividad laboral, los factores relacionados con la globalización, los cambios demográficos, la utilización masiva de la tecnología, la digitalización de un número significado de procesos, el carácter multidisciplinar del abordaje de la atención al trabajador y unos planteamientos próximos a la salud pública, determinan un nuevo enfoque de la seguridad y salud ocupacional y suscitan un debate ético. En esta realidad socioprofesional y laboral, la intervención de esta disciplina enfermera tendrá un sustrato de actuación basado en una atención integral, segura, colaborativa, coordinada, de calidad, centrada en las personas y basada en la mejor evidencia científica disponible.

Palabras clave: Enfermería del Trabajo, Salud holística.

\begin{abstract}
Occupational health nursing has a unique opportunity to identify the needs of the working population within the scope of its competences. In the different possible future of work scenarios, factors related to globalization, demographic changes, the massive use of technology, the digitization of a significant number of processes, the multidisciplinary nature of the approach to worker care and some approaches close to public health, are driving a new approach to occupational health and safety, while eliciting an ethical debate. In this social, professional and occupational reality, interventions by this nursing subspecialty will need to be based on comprehensive, safe, collaborative, coordinated, quality care, focused on people and based on the best available scientific evidence.
\end{abstract}

Key words: Occupational health Nursing, Holistic health. 
La enfermería del trabajo se encuentra en una posición privilegiada para identificar las necesidades de la población laboral en el ámbito de sus competencias. En este contexto, su presencia juega un papel activo en el aumento de la calidad de vida en el trabajo y contribuye a garantizar el bienestar de las personas ${ }^{(1)}$.

La Comisión Mundial sobre el Futuro del Trabajo propone instaurar programas centrados en las personas como práctica empresarial para el futuro de la actividad laboral(2). En el mundo del trabajo actual donde su naturaleza resulta compleja, errática y variable, los valores del binomio salud y trabajo están íntimamente relacionados. Esa interacción se expresa con nitidez en la vida laboral de las personas en distintas facetas. Si bien la salud es necesaria para desarrollar un cometido profesional de forma óptima, las condiciones de trabajo pueden perjudicar o potenciar ese bienestar de manera manifiesta ${ }^{(3,4)}$. Los efectos negativos de la relación trabajo y salud tienen en la práctica una traducción concreta en las estadísticas. La Organización Internacional del Trabajo (OIT) estima que en el mundo mueren cada día 1.000 personas debido a accidentes de trabajo y otras 6.500 a enfermedades profesionales ${ }^{(5)}$. Por tanto, el coste humano y económico aloja pocas dudas: consecuencias deletéreas para los trabajadores y su entorno, menoscabo para las empresas y el consiguiente impacto social( ${ }^{(6)}$. El impacto se cuantifica en indemnizaciones, jornadas laborales perdidas, daños materiales, sanciones administrativas, demandas por responsabilidades, interrupciones en el proceso productivo, costes asistenciales, pérdida de personal cualificado y readaptación profesional entre otras, que se estima alcanzan el 3,94\% del Producto Interior Bruto mundial cada año ${ }^{(5,7)}$. Ante esta realidad, toma más valor si cabe el pleno alcance del objetivo número ocho propuesto por la Organización de Naciones Unidas en su Agenda 2030 de Desarrollo Sostenible: promover un crecimiento económico inclusivo y sostenible junto con un trabajo decente para todos ${ }^{(8)}$.

La seguridad y salud laboral es clave para tener éxito en ese objetivo de desarroIlo sostenible. Entre otros factores que condicionan ese reto podemos citar: algunos efectos de la globalización como los cambios continuos en la organización del trabajo, reducción de las plantillas, posibilidad de automatización de tareas, deslocalización de las organizaciones y creación de pequeñas empresas donde la protección del trabajador desde el ámbito de actuación de la enfermería del trabajo apunta nuevos desafíos(4). Esta realidad refleja un escenario con unas expectativas o intereses por parte de los distintos actores sociales, la aparición de enfermedades emergentes ligadas al trabajo, el envejecimiento de los trabajadores, una incorporación de población migrante consecuencia de los movimientos demográficos, el impacto de las aplicaciones tecnológicas en las nuevas formas de trabajo ${ }^{(9)}$ y una evolución de la relación con la población laboral debido al carácter multidisciplinar en su abordaje ${ }^{(10)}$. Todo ello sin olvidar los efectos del cambio climático en la salud(7).

La situación descrita constituye una nueva apuesta en relación con la prevención. La Comisión Mundial sobre el Futuro del Trabajo de la OIT propone una visión holística ante los riesgos actuales y futuros a los que está expuesto el trabajador y los efectos adversos que provocan ${ }^{(2)}$. Esta visión integral se muestra en sintonía 
con la percepción que las enfermeras del trabajo expresan en el primer estudio sobre sus competencias profesionales en la Unión Europea, considerando este enfoque una cuestión prioritaria en la atención global al trabajador ${ }^{(11)}$. Aspecto que además se recoge en el programa formativo de la especialidad de enfermería del trabajo en España dentro las competencias de las áreas preventiva y assistencial(12) y, en el ámbito comunitario, forma parte de las conclusiones del estudio de la Federation of Occupational Health Nurses within the European Union ${ }^{(13)}$.

Los efectos y resultados de este planteamiento integral tienen una repercusión tanto en la salud y bienestar de las personas como en la sociedad en su conjunto. En un mundo donde asoman las incertidumbres y aumentan las desigualdades, distintas instituciones y autores proponen modelos de intervención holísticos que denotan aspectos comunes en su abordaje. La Organización Mundial de la Salud (OMS) aboga por una comunicación real con el ámbito comunitario a través de la incorporación a programas de salud pública durante toda la vida profesional(9). En paralelo, la OIT defiende una propuesta centrada en las personas y situada en el epicentro de las políticas económicas, sociales y de la práctica empresarial. La estrategia busca proporcionar una protección social durante todo el ciclo vital de trabajo, aplicar un programa transformador y mensurable de la igualdad de género en el ámbito de la prevención de riesgos laborales y, por último, un aumento de la inversión tecnológica en favor de un trabajo decente y sostenible ${ }^{(4)}$. En ese horizonte, el National Institute for Occupational Safety and Health (NIOSH) instaura la iniciativa Total Worker Health ${ }^{\circledR}$ con un enfoque más holístico de la salud y la seguridad de los trabajadores que trascienda de la atención exclusiva en los centros de trabajo. En definitiva, una visión más panorámica tanto de los factores relacionados con la actividad como de las circunstancias extralaborales que condicionan su bienestar ${ }^{(14,15)}$. La propia American Association of Occupational Health Nurses $(\mathrm{AAOHN})$ asume este planteamiento a través de un documento institucional de posicionamiento e invita a los profesionales a instaurar esta iniciativa de cultura del bienestar en las organizaciones ${ }^{(16)}$.

En el ámbito de la Unión Europea, el European Trade Union Institute reunió a 50 expertos en prevención de riesgos laborales y concluyeron que el futuro del trabajo se basará en cuatro escenarios: bienestar, productividad, protección social y autonomía en el desempeño de las tareas, factores que en su modelo estimulan la responsabilidad social corporativa y la participación de los empleados ${ }^{(17)}$. En paralelo, la Estrategia Europea de Salud y Seguridad en el Trabajo aboga por la colaboración entre los profesionales de la salud laboral en la identificación de riesgos nuevos y emergentes. En esta Cuarta Revolución Industrial, también conocida como Trabajo 4.0, Schulte et al. observan la necesidad de plantear un modelo expansivo tanto en una visión horizontal como vertical(18). El enfoque transversal reconoce no sólo la influencia de los riesgos estrictamente ocupacionales sino también la determinación de los factores personal, familiar, social, económico y comunitario en el impacto final de salud individual. En esta visión ampliada, la perspectiva vertical tiene presente los valores de la productividad, bienestar y el perfil multidisciplinar en la intervención, pero considera el carácter agregado de la exposición a distintos riesgos durante todo el ciclo vital y laboral, precariedad laboral, ingresos inesta- 
bles, cambios continuos de puesto de trabajo, desempleo, subempleo, etc. ${ }^{(19,20)}$. Reconociendo que todo ello tiene una repercusión relevante en el estado de salud, este modelo global también incide en tres cuestiones: la formación de los nuevos especialistas en salud laboral desde esta perspectiva, el enfoque de la investigación que analice los factores determinantes del bienestar y la forma de incorporar esta visión en las políticas empresariales. Más allá de planteamientos tradicionales relacionados con las disciplinas afines a la seguridad y salud en el trabajo, este paradigma entiende oportuno considerar las aportaciones de otras áreas como la sociología, la gerontología, las ciencias medioambientales, los expertos en recursos humanos y los especialistas en responsabilidad social corporativa(19,20). En esta propuesta integral, la enfermería del trabajo aporta experiencia y colaboración en aquellas iniciativas en favor de las personas trabajadoras.

En resumen, profundizar en los posibles escenarios futuros de la actividad laboral, los factores relacionados con la globalización, los cambios y movimientos demográficos, la utilización masiva de la tecnología, la digitalización de un número significado de procesos, el carácter multidisciplinar del abordaje de la atención al trabajador y unos planteamientos más próximos a la salud pública, constituyen el denominador común en el análisis. Estos ámbitos también invitan a plantear un debate abierto en relación con la prevención de riesgos laborales ${ }^{(8)}$. Debate que se traduce en el equilibrio de un nuevo espacio entre mantener un trabajo seguro y saludable de los empleados, la mayor productividad de las empresas y el bienestar de la sociedad(21).

El concepto más amplio de bienestar de los trabajadores se incorpora a las organizaciones a principios de este siglo, su estudio se aborda desde conceptos tan variados como la satisfacción laboral, el compromiso de los trabajadores o el estado de salud física y mental ${ }^{(14)}$. A la evaluación inicial de riesgos relacionados con la actividad profesional, se une una versión más extensa que permite incorporar la promoción de la salud y el consiguiente bienestar en el ideario de las empresas. En paralelo, la realidad sociolaboral en la Unión Europea se hace visible en un envejecimiento progresivo de sus activos: en 2009 el colectivo de trabajadores de 55 a 64 años representaba el 44\% de la población laboral, en 2019 alcanza el 59\%(22). El Foro Económico Mundial señala que las enfermedades crónicas son responsables de más de la mitad de las muertes en el mundo y prevé que represente dos tercios de todos los fallecimientos en los próximos años. Una realidad donde la incorporación de hábitos saludables se relaciona con su reducción en el 70\% de los casos ${ }^{(23)}$. Esta epidemia de enfermedades crónicas se concentró inicialmente en países desarrollados y la globalización ha contribuido a su expansión por las economías emergentes. Se estima que Brasil, China, Rusia e India pierden más de 20 millones de años productivos anuales debido a las enfermedades crónicas y en Estados Unidos la atención a este tipo de patologías representa el 75\% del gasto sanitario total(24). De forma previa, la Comisión Permanente de la International Commission on Occupational Health de la que es miembro activo la enfermería del trabajo, realizó un primer documento sobre la aportación de la especialidad a la salud de los trabajadores ya en 1969(25). Años más tarde, la propia Comisión publicó otro informe que describe la evolución de las competencias en los últimos 
treinta años. Sin abandonar su rol más tradicional basado en la atención a los accidentes de trabajo y la prestación de primeros auxilios, la especialidad tiende hacia un escenario de competencias laborales más amplio. Las actuaciones se centran en una mayor autonomía e independencia profesional, la promoción de hábitos saludables y la educación para la salud dirigida a los trabajadores ${ }^{(26)}$, intervenciones que contribuyen a atenuar la realidad señalada y que deben ser objeto de una evaluación bajo criterios de coste-eficacia.

Aunque el beneficio que reporta la mejora de la seguridad y salud en el trabajo es cada vez mayor, no resulta fácil definir una imagen concisa de su alcance global. Ante los retos descritos asoma una pregunta concreta: ¿qué se puede hacer desde el ámbito de actuación profesional de la enfermería del trabajo? La colaboración entre los actores implicados resulta primordial y más necesaria que nunca. No obstante, las líneas de investigación de los profesionales vinculados a la disciplina de la salud laboral también pueden aportar luz. Las prioridades se centran en los siguientes ámbitos: estimar el impacto económico de las iniciativas implementadas, valorar las consecuencias de los riesgos psicosociales, analizar los efectos de las aplicaciones tecnológicas y las nuevas formas de trabajo, identificar las enfermedades profesionales relacionadas con las tareas y la adaptación de los trabajadores con capacidades limitadas. También muestran interés en la reincorporación tras una ausencia prolongada, la presencia de trabajadores vulnerables o especialmente sensibles, la incorporación de trabajadores migrantes, la prolongación de la vida laboral en condiciones saludables y el cáncer ocupacional. Estas preferencias están en concordancia con las propuestas de la National Occupational Research Agenda de NIOSH${ }^{(27)}$. En cambio, concitan una menor consideración los trastornos musculoesqueléticos y en particular aquellos vinculados con el estrés laboral, la promoción de la salud en el trabajo, la gestión del absentismo y el presentismo relacionado con la enfermedad y los aspectos de género en el contexto de la prevención de riesgos laborales ${ }^{(28,29)}$.

Finalmente, en la descripción de los posibles escenarios futuros de trabajo y los modelos de análisis y actuación señalados, la enfermería del trabajo constituye un eje primordial. Las propuestas presentadas expresan unas pautas de orientación profesional con el fin de lograr una mejora en los resultados de salud de la población trabajadora. Por tanto, estas intervenciones tendrán como sustrato una atención integral, segura, colaborativa, coordinada, de calidad, centrada en las personas y basada en la mejor evidencia científica disponible ${ }^{(30,31,32)}$.

\section{Agradecimientos}

A Jordi Delclos Clanchet, Isabel López García y Alfonso Lourido Fernández, revisores de mirada elocuente. 


\section{Bibliografía}

1. González J. Enfermería del Trabajo, un valor añadido en las organizaciones. Med Segur Trab. 2019;65(254):3-9.

2. Organización Internacional del Trabajo. Trabajar para un futuro más prometedor. Comisión mundial sobre el futuro del trabajo. Ginebra: Organización Internacional del Trabajo; 2019. [Acceso: 8 Dic 2020]. Disponible en: https://www. ilo.org/wcmsp5/groups/public/---dgreports/---cabinet/documents/publication/ wcms_662442.pdf

3. Vega ML. ¿El trabajo es salud? Arch Prev Riesgos Labor. 2020;23(4):410-14. doi:10.12961/aprl.2020.23.04.01

4. European Agency for Safety and Health at Work. Third european survey of enterprises on new and emerging risks (ESENER 3). Bilbao: European Agency for Safety and Health at Work; 2019. [Acceso: 6 Nov 2020]. Disponible en: https://osha.europa.eu/es/publications/third-european-survey-enterprises-new-and-emerging-risks-esener-3/view

5. Organización Internacional del Trabajo. Seguridad y salud en el centro del futuro del trabajo. Aprovechar 100 años de experiencia. Ginebra: Organización Internacional del Trabajo; 2019. [Acceso: 6 Nov 2020]. Disponible en: https://www. ilo.org/wcmsp5/groups/public/---dgreports/---dcomm/documents/publication/ wcms_686762.pdf

6. Hämäläinen P, Takala, J, Boon Kiat, T. Global estimates of occupational accidents and work-related illnesses 2017. Finland: Workplace Safety and Health Institute; 2017. [Acceso: 23 Nov 2020]. Disponible en: http://www.icohweb.org/site/ images/news/pdf/Report\%20Global\%20Estimates\%20of\%200ccupational\%20 Accidents\%20and\%20Work-related\%20IIInesses\%202017\%20rev1.pdf

7. Organización Internacional del Trabajo. Las reglas del juego. Una introducción a la actividad normativa de la Organización Internacional del Trabajo. Ginebra: Organización Internacional del Trabajo; 2019. [Acceso: 26 Nov 2020]. Disponible en: https://www.ilo.org/wcmsp5/groups/public/---ed_norm/---normes/documents/ publication/wcms_672554.pdf

8. Naciones Unidas. La agenda 2030 y los objetivos de desarrollo sostenible. Santiago: Naciones Unidas; 2018.

9. Schulte PA, Streit JMK, Sheriff F, Delclos G, Felknor SA, Tamers SL, Fendinger S, Grosch J, Robert S. Potential scenarios and hazards in the work of the future: a systematic review of the peer-reviewed and gray literatures. Ann Work Expo Health. 2020;1-31. doi:10.1093/annweh/wxaa051.

10. World Health Organization. European programme of work 2020-2025. United action for better health in Europe. Copenhagen: World Health Organization; 2020. [Acceso: 18 Nov 2020]. Disponible en: https://apps.who.int/iris/bitstream/handle/10665/333908/70wd11e-rev4-EPW 200673. pdf? sequence=1\&isAllowed=y 
11. González J. Estudio sobre las competencias profesionales de enfermería del trabajo en España. Arch Prev Riesgos Labor. 2020;23(1):34-51. doi:10.12961/ aprl.2020.23.01.4.

12. Boletín Oficial del Estado. Orden SAS/1348/2009 de 6 de mayo por la que se aprueba y publica el programa formativo de la especialidad de Enfermería del Trabajo. BOE núm. 129 de 28/5/2009. [Acceso: 20 Nov 2020]. Disponible en: http:// www.boe.es/boe/dias/2009/05/28/pdfs/BOE-A-2009-8880.pdf

13. Staun J. Occupational Health Nurses status in European Union states 20052012. Final results. Federation of Occupational Health Nurses within the European Union, 2014.

14. Chari R, Chang CC, Sauter SL, Petrun Sayers EL, Cerully JL, Schulte P, Schill AL, Uscher-Pines L. Expanding the paradigm of occupational safety and health: a new framework for worker well-being. J Occup Environ Med. 2018;60(7):589-593.

15. National Institute for Occupational Safety and Health. Fundamentals of Total Worker Health ${ }^{\circledR}$ approaches. Essential elements for advancing worker safety, health and well-being. Atlanta: National Institute for Occupational Safety and Health; 2016. [Acceso: 2 Nov 2020]. Disponible en: https://www.cdc.gov/niosh/ docs/2017-112/pdfs/2017_112.pdf

16. American Association of Occupational Health Nurses. Health promotion and productivity in the workplace: the occupational and environmental health nurse role in supporting the workforce using NIOSH's Total Worker Health ${ }^{\circledR}$ approach. Workplace Health Saf. 2020;11(6). doi:10.1177/2165079920967808.

17. European Trade Union Institute. Occupational safety and health in 2040 . four scenarios. How will the next generation in Europe deal with occupational safety and health issues? Brussels: European Trade Union Institute; 2017. [Acceso: 20 Dic 2020]. Disponible en: https://www.etui.org/sites/default/files/Guide_OSH_web.pdf

18. European Commission. Communication from the Commission to the European Parliament, The Council, The European Economic and Social Committee and The Committee of the Regions on an EU Strategic Framework on Health and Safety at Work 2014-2020. Brussels: European Commission; 2014. [Acceso: 20 Dic 2020]. Disponible en: https://eur-lex.europa.eu/legal-content/EN/TXT/PDF/?uri=CELEX:52014DC0332.

19. Schulte PA, Delclos G, Felknor SA, Chosewood LC. Toward an expanded focus for occupational safety and health: a commentary. Int J Environ Res Public Health. 2019;16(24):4946. doi:10.3390/ijerph16244946.

20. Schulte P, Vainio H. Well-being at work. Overview and perspective. Scand J Work Environ Health. 2010;36(5):422-29. doi:10.5271/sjweh.3076.

21. lavicoli S, Valenti A, Gagliardi D, Rantanen J. Ethics and occupational health in the contemporary world of work. Int J Environ Res Public Health. 2018;15(8):1713. doi:10.3390/ijerph15081713. 
22. European Commission. European Commission report on the impact of demographic change. Brussels: European Commission; 2020. [Acceso: 2 Dic 2020]. Disponible en: https://ec.europa.eu/info/sites/info/files/demography_report_2020_n. pdf

23. World Economic Forum. The new discipline of workforce wellness. Enhancing corporate performance by tackling chronic disease. Geneva: World Economic Forum; 2010. [Acceso: 9 Dic 2020]. Disponible en: http://www3.weforum.org/ docs/WEF_HE_TacklingChronicDisease_Report_2010.pdf

24. World Economic Forum. Working towards wellness: accelerating the prevention of chronic disease. Geneva: World Economic Forum; 2018. [Acceso: 20 Dic 2020]. Disponible en: https://www.pwc.pl/en/publikacje/business_rationale.pdf

25. Permanent Commission and International Association on Occupational Health. Report of the nursing sub-committee on the nurse's contribution to the health of the worker 1966-1969. London: Permanent Commission and International Association on Occupational Health; 1969.

26. Burgel B, Camp J, Lepping G. The nurse's contribution to the health of the worker. Occupational Health Nursing in 2000. An international perspective. Scientific Committee on Occupational Health Nursing Working Party Report 10; 2005. [Acceso: 4 Dic 2020]. Disponible en: http://www.icohweb.org/site/multimedia/scientific_committees/pdf/SCOHNursing.pdf

27. Wachs JE. The American Association of Occupational Health Nurses. Seventy-five years of education, practice and research. Workplace Health Saf. 2017;65(4):148-153. doi:10.1177/2165079917701139.

28. Gagliardi D, Rondinone BM, Mirabile, Buresti G, Ellwood P, Hery M, Paszkiewicz P, Valenti A, lavicoli S. The perspective of European researchers of national occupational safety and health institutes for contributing to a European research agenda: a modified Delphi study. BMJ Open. 2017;7:e015336. doi:10.1136/ bmjopen-2016-015336.

29. Lalloo D, Demou E, Smedley J, Madan I, Asanati K, Macdonald EB. Current research priorities for UK occupational physicians and occupational health researchers: a modified Delphi study. Occup Environ Med. 2018;75(11):830-6. doi:10.1136/oemed-2018-105114.

30. Boletín Oficial del Estado. Resolución de 20 de octubre de 2020 de la Dirección General de Salud Pública por la que se valida la Guía para la indicación, uso y autorización de dispensación de medicamentos sujetos a prescripción médica por parte de las/los enfermeras/os de heridas. BOE núm. 286 de 29/10/2020. [Acceso: 1 Dic 2020]. Disponible en: https://www.boe.es/boe/dias/2020/10/29/pdfs/ BOE-A-2020-13190.pdf

31. Diario Oficial de la Unión Europea. Directiva 2013/55/UE del Parlamento Europeo y del Consejo de 20 de noviembre de 2013 por la que se modifica la Directiva 2005/36/CE relativa al reconocimiento de cualificaciones profesionales y el Reglamento UE núm. 1024/2012 relativo a la cooperación administrativa a través 
del Sistema de Información del Mercado Interior (Reglamento IMI). DOCE núm. L 354/132 de 28/12/2013. [Acceso: 2 Feb 2021]. Disponible en: https://www.boe.es/ doue/2013/354/L00132-00170.pdf

32. Boletín Oficial del Estado. Real Decreto $581 / 2017$ de 9 de junio por el que se incorpora al ordenamiento jurídico español la Directiva 2013/55/UE del Parlamento Europeo y del Consejo de 20 de noviembre de 2013 por la que se modifica la Directiva 2005/36/CE relativa al reconocimiento de cualificaciones profesionales y el Reglamento UE núm. 1024/2012 relativo a la cooperación administrativa a través del Sistema de Información del Mercado Interior (Reglamento IMI). BOE núm. 138 de 10/6/2017. [Acceso: 2 Feb 2021]. Disponible en: https://www.boe.es/buscar/ pdf/2017/BOE-A-2017-6586-consolidado.pdf 